\title{
Relaxed resolvent operator for solving a variational inclusion problem
}

\author{
Iqbal Ahmad, Mijanur Rahaman, Rais Ahmad* \\ Department of Mathematics, Aligarh Muslim University, India
}

(Received: 5 September 2015; Accepted: 7 May 2016)

\begin{abstract}
In this paper, we introduce a new resolvent operator and we call it relaxed resolvent operator. We prove that relaxed resolvent operator is single-valued and Lipschitz continuous and finally we approximate the solution of a variational inclusion problem in Hilbert spaces by defining an iterative algorithm based on relaxed resolvent operator. A few concepts like Lipschitz continuity and strong monotonicity are used to prove the main result of this paper. Thus, no strong conditions are used. Some examples are constructed.
\end{abstract}

Keywords Relaxed; Inclusion; Space; Algorithm; Lipschitz

AMS 2010 subject classifications 40J40; 47H19

DOI: $10.19139 /$ soic.v4i2.166

\section{Introduction and Preliminaries}

Variational inequality is an inequality involving a functional, which has to be solved for all possible values of a given variable, belonging usually to a convex set. The theory of variational inequalities was initially developed to deal with equilibrium problems, precisely the Signorini problem. After that it has been extended and generalized to study a wide class of problems arising in mechanics, physics, optimization and control, nonlinear programming, economics, finance, regional structural,transformation, elasticity, and applied sciences, etc., see e.g., $[1,2,4,12,15,16,17,18,19,20]$ and references therein.

A useful and important generalization of variational inequalities is called a variational inclusion which was introduced by Hassouni and Moudafi [10] and includes mixed variational inequalities as special cases. Many problems concerning variational inclusions are solved by using the concept of maximal monotonicity and its generalized concepts such as $H$-monotonicity [6], $H$-accretivity [5] etc., see e.g., [7, 8, 9, 13, 21] and references therein. Most of the splitting methods are based on the resolvent operator of the form $[I+\lambda M]^{-1}$, where $M$ is a set-valued monotone mapping, $\lambda$ is a positive constant and $I$ is the identity mapping.

In this paper, we introduce a new resolvent operator of the form $[(I-H)+\lambda M]^{-1}$, where $H$ is a relaxed Lipschitz continuous mapping, $M$ is a set-valued monotone mapping, $\lambda$ is a positive constant and $I$ is an identity mapping. We call this new resolvent operator as relaxed resolvent operator and prove that it is single-valued and Lipschitz continuous. We define an iterative algorithm based on relaxed resolvent operator to solve a variational inclusion problem. Convergence of the iterative sequences generated by the iterative algorithm is also discussed. Some examples are constructed.

\footnotetext{
*Correspondence to: Rais Ahmad (Email: raisain_123@rediffmail.com). Department of Mathematics, Aligarh Muslim University, Aligarh
} 202002, India

ISSN 2310-5070 (online) ISSN 2311-004X (print)

Copyright (C) 2016 International Academic Press 
Throughout this paper, we suppose that $X$ is a real Hilbert space endowed with a norm $\|\cdot\|$ and an inner product $\langle\cdot, \cdot\rangle, d$ is the metric induced by the norm $\|\cdot\|, 2^{X}$ (respectively, $C B(X)$ ) is the family of all nonempty (respectively, closed and bounded) subsets of $X$, and $D(\cdot, \cdot)$ is the Hausdorff metric on $C B(X)$ defined by

$$
D(P, Q)=\max \left\{\sup _{x \in P} d(x, Q), \sup _{y \in Q} d(P, y)\right\},
$$

where $d(x, Q)=\inf _{y \in Q} d(x, y)$ and $d(P, y)=\inf _{x \in P} d(x, y)$.

The following definitions are needed in the sequel.

\section{Definition 1.1}

A mapping $g: X \rightarrow X$ is said to be

(i) Lipschitz continuous if, there exist a constant $\lambda_{g}>0$ such that

$$
\|g(x)-g(y)\| \leq \lambda_{g}\|x-y\|, \forall x, y \in X
$$

(ii) monotone, if

$$
\langle g(x)-g(y), x-y\rangle \geq 0, \forall x, y \in X
$$

(iii) strongly monotone if, there exists a constant $\xi>0$ such that

$$
\langle g(x)-g(y), x-y\rangle \geq \xi\|x-y\|^{2}, \forall x, y \in X
$$

(iv) relaxed Lipschitz continuous if, there exists a constant $r>0$ such that

$$
\langle g(x)-g(y), x-y\rangle \leq-r\|x-y\|^{2}, \forall x, y \in X .
$$

\section{Definition 1.2}

A mapping $N: X \times X \times X \rightarrow X$ is said to be Lipschitz continuous with respect to first argument if, there exists a constant $\lambda_{N_{1}}$ such that

$$
\left\|N\left(x_{1}, x_{2}, x_{3}\right)-N\left(y_{1}, x_{2}, x_{3}\right)\right\| \leq \lambda_{N_{1}}\left\|x_{1}-y_{1}\right\|, \forall x_{1}, y_{1}, x_{2}, x_{3} \in X .
$$

Similarly, we can define the Lipschitz continuity of $N$ in rest of the arguments.

\section{Definition 1.3}

A set-valued mapping $A: X \rightarrow C B(X)$ is said to be $D$-Lipschitz continuous if, there exists a constant $\delta_{A}$ such that

$$
D(A(x), A(y)) \leq \delta_{A}\|x-y\|, \forall x, y \in X .
$$

\section{Relaxed Resolvent operator}

We begin this section with the introduction of relaxed resolvent operator and demonstrate some of its properties.

\section{Definition 2.1}

Let $H: X \rightarrow X$ be a mapping and $I: X \rightarrow X$ be an identity mapping. Then, a set-valued mapping $M: X \rightarrow 2^{X}$ is a said to be $(I-H)$-monotone if, $M$ is monotone, $H$ is relaxed Lipschitz continuous and

$$
[(I-H)+\lambda M](X)=X,
$$

where $\lambda>0$ is a constant. 
Definition 2.2

Let $H: X \rightarrow X$ be relaxed Lipschitz continuous mapping and $I: X \rightarrow X$ be an identity mapping. Suppose that $M: X \rightarrow 2^{X}$ is a set-valued, $(I-H)$-monotone mapping. The relaxed resolvent operator $R_{\lambda, M}^{(I-H)}: X \rightarrow X$ associated with $I, H$ and $M$ is defined by

$$
R_{\lambda, M}^{I-H}(x)=[(I-H)+\lambda M]^{-1}(x), \forall x \in X,
$$

where $\lambda>0$ is a constant.

\section{Example 2.1}

Let $X=S^{2}$, the space of all $2 \times 2$ real symmetric matrices equipped with inner product $\langle A, B\rangle=\operatorname{tr}(A \cdot B)$, $\forall A, B \in X$, and let $\alpha, \beta$ be two positive real numbers such that $\beta \leq \alpha$. Let $H: X \rightarrow X$ be a mapping defined by

$$
H\left(\left[\begin{array}{cc}
x_{1} & a \\
a & x_{2}
\end{array}\right]\right)=\left[\begin{array}{cc}
-\alpha x_{1} & a \\
a & -\alpha x_{2}
\end{array}\right], \forall x_{1}, x_{2}, a \in \mathbb{R}
$$

For $x=\left[\begin{array}{cc}x_{1} & a \\ a & x_{2}\end{array}\right] \in X$, we calculate,

$$
\begin{aligned}
& \langle H(x)-H(y), x-y\rangle \\
= & \left\langle\left[\begin{array}{cc}
-\alpha\left(x_{1}-y_{1}\right) & 0 \\
0 & -\alpha\left(x_{2}-y_{2}\right)
\end{array}\right],\left[\begin{array}{cc}
\left(x_{1}-y_{1}\right) & 0 \\
0 & \left(x_{2}-y_{2}\right)
\end{array}\right]\right\rangle \\
= & \operatorname{tr}\left(\left[\begin{array}{cc}
-\alpha\left(x_{1}-y_{1}\right) & 0 \\
0 & -\alpha\left(x_{2}-y_{2}\right)
\end{array}\right] \cdot\left[\begin{array}{cc}
\left(x_{1}-y_{1}\right) & 0 \\
0 & \left(x_{2}-y_{2}\right)
\end{array}\right]\right) \\
= & -\alpha\left(x_{1}-y_{1}\right)^{2}-\alpha\left(x_{2}-y_{2}\right)^{2} \\
= & -\alpha\left[\left(x_{1}-y_{1}\right)^{2}+\left(x_{2}-y_{2}\right)^{2}\right] \\
\leq & -\beta\left[\left(x_{1}-y_{1}\right)^{2}+\left(x_{2}-y_{2}\right)^{2}\right] .
\end{aligned}
$$

Also,

$$
\begin{aligned}
\|x-y\|^{2} & =\langle x-y, x-y\rangle \\
& =\left\langle\left[\begin{array}{cc}
x_{1}-y_{1} & 0 \\
0 & x_{2}-y_{2}
\end{array}\right],\left[\begin{array}{cc}
x_{1}-y_{1} & 0 \\
0 & x_{2}-y_{2}
\end{array}\right]\right\rangle \\
& =\operatorname{tr}\left(\left[\begin{array}{cc}
x_{1}-y_{1} & 0 \\
0 & x_{2}-y_{2}
\end{array}\right] \cdot\left[\begin{array}{cc}
x_{1}-y_{1} & 0 \\
0 & x_{2}-y_{2}
\end{array}\right]\right) \\
& =\left(x_{1}-y_{1}\right)^{2}+\left(x_{2}-y_{2}\right)^{2} .
\end{aligned}
$$

From above it follows that

$$
\langle H(x)-H(y), x-y\rangle \leq-\beta\|x-y\|^{2}, \forall x, y \in X .
$$

i.e., $H$ is $\beta$-relaxed Lipschitz continuous.

Suppose that $M: X \rightarrow 2^{X}$ is defined by

$$
M\left(\left[\begin{array}{cc}
x_{1} & a \\
a & x_{2}
\end{array}\right]\right)=\left[\begin{array}{cc}
\alpha x_{1} & a \\
a & \alpha x_{2}
\end{array}\right], \forall x_{1}, x_{2}, a \in \mathbb{R} .
$$


We calculate

$$
\begin{aligned}
& \langle M(x)-M(y), x-y\rangle \\
= & \left\langle M\left(\left[\begin{array}{cc}
x_{1} & a \\
a & x_{2}
\end{array}\right]\right)-M\left(\left[\begin{array}{cc}
y_{1} & a \\
a & y_{2}
\end{array}\right]\right),\left[\begin{array}{cc}
x_{1}-y_{1} & 0 \\
0 & x_{2}-y_{2}
\end{array}\right]\right\rangle \\
= & \left\langle\left[\begin{array}{cc}
\alpha\left(x_{1}-y_{1}\right) & 0 \\
0 & \alpha\left(x_{2}-y_{2}\right)
\end{array}\right],\left[\begin{array}{cc}
x_{1}-y_{1} & 0 \\
0 & x_{2}-y_{2}
\end{array}\right]\right\rangle \\
= & \operatorname{tr}\left(\left[\begin{array}{cc}
\alpha\left(x_{1}-y_{1}\right) & 0 \\
0 & \alpha\left(x_{2}-y_{2}\right)
\end{array}\right] \cdot\left[\begin{array}{cc}
x_{1}-y_{1} & 0 \\
0 & x_{2}-y_{2}
\end{array}\right]\right) \\
= & \alpha\left(x_{1}-y_{1}\right)^{2}+\alpha\left(x_{2}-y_{2}\right)^{2},
\end{aligned}
$$

which implies that

$$
\langle M(x)-M(y), x-y\rangle \geq 0, \forall x, y \in X
$$

i.e., $M$ is monotone.

Now, we show that for $\lambda=1$, every element $x=\left[\begin{array}{cc}\alpha x_{1} & a \\ a & \alpha x_{2}\end{array}\right] \in 2^{X}$ has a pre-image $y=\left[\begin{array}{cc}\frac{\alpha x_{1}}{1+2 \alpha} & a \\ a & \frac{\alpha x_{2}}{1+2 \alpha}\end{array}\right] \in$ $X$.

$$
\begin{aligned}
{[(I-H)+M](y) } & =(I-H)(y)+M(y) \\
& =y-H(y)+M(y) \\
& =\left[\begin{array}{cc}
\frac{\alpha x_{1}}{1+2 \alpha} & a \\
a & \frac{\alpha x_{2}}{1+2 \alpha}
\end{array}\right]-\left[\begin{array}{cc}
\frac{-\alpha^{2} x_{1}}{1+2 \alpha} & a \\
a & \frac{-\alpha^{2} x_{2}}{1+2 \alpha}
\end{array}\right]+\left[\begin{array}{cc}
\frac{\alpha^{2} x_{1}}{1+2 \alpha} & a \\
a & \frac{\alpha^{2} x_{2}}{1+2 \alpha}
\end{array}\right] \\
& =\left[\begin{array}{cc}
\frac{\alpha(1+2 \alpha) x_{1}}{1+2 \alpha} & a \\
a & \frac{\alpha(1+2 \alpha) x_{2}}{1+2 \alpha}
\end{array}\right] \\
& =\left[\begin{array}{cc}
\alpha x_{1} & a \\
a & \alpha x_{2}
\end{array}\right] \in 2^{X} .
\end{aligned}
$$

It follows that

$$
[(I-H)+M](X)=X
$$

i.e., $M$ is $(I-H)$-monotone mapping.

Now, we prove some of the properties of relaxed resolvent operator defined by (1).

Theorem 2.2

Let $H: X \rightarrow X$ be a $r$-relaxed Lipschitz continuous mapping, $I: X \rightarrow X$ be an identity mapping and $M: X \rightarrow$ $2^{X}$ be a set-valued $(I-H)$-monotone mapping. Then the operator $[(I-H)+\lambda M]^{-1}$ is single-valued, where $\lambda>0$ is a constant.

Proof

For any $z \in X$ and a constant $\lambda>0$, let $x, y \in[(I-H)+\lambda M]^{-1}(z)$. Then,

$$
\begin{aligned}
& \lambda^{-1}[z-(I-H)(x)] \in M(x) ; \\
& \lambda^{-1}[z-(I-H)(y)] \in M(y) .
\end{aligned}
$$


Since $M$ is monotone, we have

$$
\begin{aligned}
\langle-(I-H)(x)+z+(I-H)(y)-z, x-y\rangle & \geq 0 ; \\
-\langle(I-H)(x)-(I-H)(y), x-y\rangle & \geq 0 ; \\
-\langle x-H(x)-y+H(y), x-y\rangle & \geq 0 ; \\
\langle x-H(x)-y+H(y), x-y\rangle & \leq 0 ; \\
\langle x-H(x)-y+H(y), x-y\rangle & \leq 0 ; \\
\langle x-y, x-y\rangle-\langle H(x)-H(y), x-y\rangle & \leq 0 .
\end{aligned}
$$

Since $H$ is $r$-relaxed Lipschitz continuous, we have

$$
0 \geq\langle x-y, x-y\rangle-\langle H(x)-H(y), x-y\rangle \geq\|x-y\|^{2}+r\|x-y\|^{2} \geq 0,
$$

it follows that $(1+r)\|x-y\|^{2}=0$, which implies that $x=y$. Thus $[(I-H)+\lambda M]^{-1}$ is single-valued.

\section{Theorem 2.3}

Let $H: X \rightarrow X$ be a $r$-relaxed Lipschitz continuous mapping, $I: X \rightarrow X$ be an identity mapping and $M: X \rightarrow$ $2^{X}$ be a set-valued, $(I-H)$-monotone mapping. Then the resolvent operator $R_{\lambda, M}^{I-H}: X \rightarrow X$ is $\frac{1}{[1+r]}$-Lipschitz continuous, i.e.,

$$
\left\|R_{\lambda, M}^{I-H}(x)-R_{\lambda, M}^{I-H}(y)\right\| \leq \frac{1}{[1+r]}\|x-y\|, \forall x, y \in X .
$$

Proof

Let $x$ and $y$ be any given point in $X$. If follow from (1) that

$$
\begin{aligned}
& R_{\lambda, M}^{I-H}(x)=[(I-H)+\lambda M]^{-1}(x), \\
& R_{\lambda, M}^{I-H}(y)=[(I-H)+\lambda M]^{-1}(y) .
\end{aligned}
$$

It follows that

$$
\begin{aligned}
& \frac{1}{\lambda}\left[x-(I-H)\left(R_{\lambda, M}^{I-H}(x)\right)\right] \in M\left(R_{\lambda, M}^{I-H}(x)\right), \\
& \frac{1}{\lambda}\left[y-(I-H)\left(R_{\lambda, M}^{I-H}(y)\right)\right] \in M\left(R_{\lambda, M}^{I-H}(y)\right) .
\end{aligned}
$$

Since $M$ is $(I-H)$-monotone i.e., $M$ is monotone, we have

$$
\begin{aligned}
& \frac{1}{\lambda}\left\langle x-(I-H)\left(R_{\lambda, M}^{I-H}(x)\right)-\left(y-(I-H)\left(R_{\lambda, M}^{I-H}(y)\right)\right), R_{\lambda, M}^{I-H}(x)-R_{\lambda, M}^{I-H}(y)\right\rangle \geq 0, \\
& \frac{1}{\lambda}\left\langle x-y-\left\{(I-H)\left(R_{\lambda, M}^{I-H}(x)\right)-(I-H)\left(R_{\lambda, M}^{I-H}(y)\right)\right\}, R_{\lambda, M}^{I-H}(x)-R_{\lambda, M}^{I-H}(y)\right\rangle \geq 0 .
\end{aligned}
$$

It follows that

$$
\begin{aligned}
& \left\langle x-y, R_{\lambda, M}^{I-H}(x)-R_{\lambda, M}^{I-H}(y)\right\rangle \\
\geq & \left\langle(I-H)\left(R_{\lambda, M}^{I-H}(x)\right)-(I-H)\left(R_{\lambda, M}^{I-H}(y)\right), R_{\lambda, M}^{I-H}(x)-R_{\lambda, M}^{I-H}(y)\right\rangle .
\end{aligned}
$$


By Cauchy-Schwartz inequality, (5) and $r$-relaxed Lipschitz continuity of $H$, we have

$$
\begin{aligned}
& \|x-y\|\left\|R_{\lambda, M}^{I-H}(x)-R_{\lambda, M}^{I-H}(y)\right\| \\
\geq & \left\langle x-y, R_{\lambda, M}^{I-H}(x)-R_{\lambda, M}^{I-H}(y)\right\rangle \\
\geq & \left\langle R_{\lambda, M}^{I-H}(x)-H\left(R_{\lambda, M}^{I-H}(x)\right)-R_{\lambda, M}^{I-H}(y)+H\left(R_{\lambda, M}^{I-H}(y)\right), R_{\lambda, M}^{I-H}(x)-R_{\lambda, M}^{I-H}(y)\right\rangle \\
= & \left\langle R_{\lambda, M}^{I-H}(x)-R_{\lambda, M}^{I-H}(y), R_{\lambda, M}^{I-H}(x)-R_{\lambda, M}^{I-H}(y)\right\rangle- \\
& \left\langle H\left(R_{\lambda, M}^{I-H}(x)\right)-H\left(R_{\lambda, M}^{I-H}(y)\right), R_{\lambda, M}^{I-H}(x)-R_{\lambda, M}^{I-H}(y)\right\rangle \\
\geq & \left\|R_{\lambda, M}^{I-H}(x)-R_{\lambda, M}^{I-H}(y)\right\|^{2}+r\left\|R_{\lambda, M}^{I-H}(x)-R_{\lambda, M}^{I-H}(y)\right\|^{2} \\
= & (1+r)\left\|R_{\lambda, M}^{I-H}(x)-R_{\lambda, M}^{I-H}(y)\right\|^{2} .
\end{aligned}
$$

Thus, we have

$$
\left\|R_{\lambda, M}^{I-H}(x)-R_{\lambda, M}^{I-H}(y)\right\| \leq \frac{1}{[1+r]}\|x-y\|,
$$

i.e., the relaxed resolvent operator $R_{\lambda, M}^{I-H}$ is $\frac{1}{[1+r]}$-Lipschitz continuous.

In support of Theorem 2.2, we have the following example.

Example 2.4

Let $X=\mathbb{R}^{2}$ with usual inner product. Let $H: X \rightarrow X$ be a mapping defined by

$$
H(x)=\left(-2 x_{1},-4 x_{2}\right), \forall x=\left(x_{1}, x_{2}\right) \in X,
$$

and the mapping $M: X \rightarrow 2^{X}$ be defined by

$$
M(x)=\left(4 x_{1}, 2 x_{2}\right), \forall x=\left(x_{1}, x_{2}\right) \in X .
$$

Then, it easy to check that $H$ is 2-relaxed Lipschitz continuous and $M$ is monotone. In addition, it is easy to verify that for $\lambda=1,[(I-H)+\lambda M](X)=X$, which shows that $M$ is $(I-H)$-monotone mapping. Hence, the relaxed resolvent operator $R_{\lambda, M}^{(I-H)}: X \rightarrow X$ associated with $I, H$ and $M$ is of the form:

$$
R_{\lambda, M}^{I-H}(x)=\left(\frac{x_{1}}{7}, \frac{x_{2}}{7}\right), \forall x=\left(x_{1}, x_{2}\right) \in X .
$$

It is easy to see that the relaxed resolvent operator defined by (2.7) is single-valued.

Now, we prove that $R_{\lambda, M}^{I-H}$ is Lipschitz continuous.

$$
\begin{aligned}
\left\|R_{\lambda, M}^{I-H}(x)-R_{\lambda, M}^{I-H}(y)\right\| & =\left\|\left(\frac{x_{1}}{7}, \frac{x_{2}}{7}\right)-\left(\frac{y_{1}}{7}, \frac{y_{2}}{7}\right)\right\| \\
& =\left\|\left(\frac{x_{1}-y_{1}}{7}, \frac{x_{2}-y_{2}}{7}\right)\right\| \\
& =\left[\frac{\left(x_{1}-y_{1}\right)^{2}}{49}+\frac{\left(x_{2}-y_{2}\right)^{2}}{49}\right]^{\frac{1}{2}} \\
& =\frac{1}{7}\left[\left(x_{1}-y_{1}\right)^{2}+\left(x_{2}-y_{2}\right)^{2}\right]^{\frac{1}{2}} \\
& \leq \frac{1}{3}\|x-y\| .
\end{aligned}
$$

Hence, the resolvent operator $R_{\lambda, M}^{I-H}$ is $\frac{1}{3}$-Lipschitz continuous. 


\section{Formulation of the problem and iterative algorithm}

In this section, we formulate a variational inclusion problem and an iterative algorithm based on relaxed resolvent operator to approximate the solution of our problem.

Let $X$ be a real Hilbert space and $H, g: X \rightarrow X, N: X \times X \times X \rightarrow X$ be the single-valued mappings, $I: X \rightarrow X$ be an identity mapping. Suppose that $A, B, C: X \rightarrow C B(X)$ and $M: X \times X \rightarrow 2^{X}$ are the set-valued mappings such that $M$ is $(I-H)$-monotone. We consider the problem of finding $x \in X, u \in A(x), v \in B(x)$, $w \in C(x)$ and $g(x) \bigcap \operatorname{dom} M(., x) \neq \emptyset$ such that

$$
0 \in N(u, v, w)+M(g(x), x) .
$$

When $C \equiv 0, N(u, v,)=.N(u, v)$, then problem (8) reduces to find $x \in X, u \in A(x), v \in B(x)$ such that

$$
0 \in N(u, v)+M(g(x), x) .
$$

Problem (9) was introduced and studied by Kazmi and Khan [11].

In addition if $M(g(x), x)=M(g(x))$, then a similar analogue of problem (9) was introduced and studied by Chang, Cho, Lee and Jung [3], Chang [2], Chang, Jim and Kim [4].

It is clear that for suitable choices of mappings involved in the formulation of problem (8), one can obtain many variational inclusion problems studied in recent past.

By applying the relaxed resolvent operator, we establish an equivalence result for variational inclusion problem (8) and a nonlinear equation.

Lemma 3.1

Let $x \in X, u \in A(x), v \in B(x)$ and $w \in C(x)$ is a solution of variational inclusion problem (3.1) if and only if $(x, u, v, w)$ satisfies the equation:

$$
g(x)=R_{\lambda, M(., x)}^{I-H}[(I-H) g(x)-\lambda N(u, v, w)],
$$

where

$$
R_{\lambda, M(., x)}^{I-H}=[(I-H)+\lambda M(., x)]^{-1},
$$

and $\lambda>0$ is a constant.

Proof

The proof is a direct consequence of Definition 2.2.

Theorem 3.2 (Nadler's Theorem [14])

Let $(X, d)$ be a complete metric space. If $F: X \rightarrow C B(X)$ is a set-valued contraction mapping, then $F$ has a fixed point.

Based on Lemma 3.1 and Theorem 3.2, we construct an iterative algorithm for finding approximate solutions of problem (8).

Iterative Algorithm 3.1

Let $g, H: X \rightarrow X, N: X \times X \times X \rightarrow X$ are the single-valued mappings, $I: X \rightarrow X$ be an identity mapping, and $A, B, C: X \rightarrow C B(X)$ are the set-valued mappings be such that for each $x \in X, Q\left(x_{0}\right) \subseteq g(x)$, where $Q: X \rightarrow 2^{X}$ be a set-valued mapping defined by

$$
Q(x)=\bigcup_{u \in A(x)} \bigcup_{v \in B(x)} \bigcup_{w \in C(x)}\left[R_{\lambda, M(., x)}^{I-H}[(I-H) g(x)-\lambda N(u, v, w)]\right]
$$

where $M: X \times X \rightarrow 2^{X}$ be a set-valued mapping such that a fixed $x \in X, M(., x)$ is $(I-H)$-monotone. 
For any given $x_{0} \in X, u_{0} \in A\left(x_{0}\right), v_{0} \in B\left(x_{0}\right)$, and $w_{0} \in C\left(x_{0}\right)$, let

$$
z_{0}=R_{\lambda, M\left(., x_{0}\right)}^{I-H}\left[(I-H) g\left(x_{0}\right)-\lambda N\left(u_{0}, v_{0}, w_{0}\right)\right] \subseteq Q\left(x_{0}\right) \subseteq g(X) .
$$

Hence, there exist $x_{1} \in X$ such that $z_{0}=g\left(x_{1}\right)$. Since $u_{0} \in A\left(x_{0}\right) \in C B(X), v_{0} \in B\left(x_{0}\right) \in C B(X)$ and $w_{0} \in$ $C\left(x_{0}\right) \in C B(X)$, by Theorem 3.2 there exist $u_{1} \in A\left(x_{1}\right), v_{1} \in B\left(x_{1}\right)$ and $w_{1} \in C\left(x_{1}\right)$ such that

$$
\begin{aligned}
\left\|u_{1}-u_{0}\right\| & \leq D\left(A\left(x_{1}\right), A\left(x_{0}\right)\right), \\
\left\|v_{1}-v_{0}\right\| & \leq D\left(B\left(x_{1}\right), B\left(x_{0}\right)\right), \\
\left\|w_{1}-w_{0}\right\| & \leq D\left(C\left(x_{1}\right), C\left(x_{0}\right)\right) .
\end{aligned}
$$

Let

$$
z_{1}=R_{\lambda, M\left(., x_{1}\right)}^{I-H}\left[(I-H) g\left(x_{1}\right)-\lambda N\left(u_{1}, v_{1}, w_{1}\right)\right] \subseteq Q\left(x_{1}\right) \subseteq g(X) .
$$

Hence, there exist $x_{2} \in X$ such that $z_{1}=g\left(x_{2}\right)$. Continuing the above process inductively, we can define the iterative sequences $\left\{x_{n}\right\},\left\{u_{n}\right\},\left\{v_{n}\right\}$ and $\left\{w_{n}\right\}$ by the following scheme:

$$
\begin{gathered}
g\left(x_{n+1}\right)=R_{\lambda, M\left(., x_{n}\right)}^{I-H}\left[(I-H) g\left(x_{n}\right)-\lambda N\left(u_{n}, v_{n}, w_{n}\right)\right], \\
u_{n+1} \in A\left(x_{n+1}\right),\left\|u_{n+1}-u_{n}\right\| \leq D\left(A\left(x_{n+1}\right), A\left(x_{n}\right)\right), \\
v_{n+1} \in B\left(x_{n+1}\right),\left\|v_{n+1}-v_{n}\right\| \leq D\left(B\left(x_{n+1}\right), B\left(x_{n}\right)\right), \\
w_{n+1} \in C\left(x_{n+1}\right),\left\|w_{n+1}-w_{n}\right\| \leq D\left(C\left(x_{n+1}\right), C\left(x_{n}\right)\right),
\end{gathered}
$$

where $\lambda>0$ is a constant and $n=0,1,2, \cdots$.

\section{Existence and convergence result}

In this section, we prove an existence and convergence result for variational inclusion problem (8).

\section{Theorem 4.1}

Let $X$ be a real Hilbert space and $g, H: X \rightarrow X$ be the single-valued mappings such that $g$ is strongly monotone with constant $\xi$, Lipschitz continuous with constant $\lambda_{g}$ and $H$ is relaxed Lipschitz continuous with constant $r$ and Lipschitz continuous with constant $\lambda_{H}$. Suppose that $N: X \times X \times X \rightarrow X$ is a single-valued mapping such that $N$ is Lipschitz continuous in all the three arguments with constants $\lambda_{N_{1}}, \lambda_{N_{2}}$ and $\lambda_{N_{3}}$, respectively and $A, B, C: X \rightarrow C B(X)$ be the set-valued mappings such that $A$ is $D$-Lipschitz continuous with constant $\lambda_{A}, B$ is $D$-Lipschitz continuous with constant $\delta_{B}$ and $C$ is $D$-Lipschitz continuous with constant $\delta_{C}$. Suppose that setvalued mapping $M: X \times X \rightarrow 2^{X}$ is such that for a fixed $x \in X, M(., x)$ is $(I-H)$-monotone with respect to the first arguments, where $I: X \rightarrow X$ is the identity mapping and for each $x \in X, Q(x) \subseteq g(x)$, where $Q$ is defined by (11). Suppose that there exists constants $\lambda>0$ and $h>0$ such that the following conditions hold:

$$
\left\|R_{\lambda, M(\cdot, x)}^{I-H}(z)-R_{\lambda, M(\cdot, y)}^{I-H}(z)\right\| \leq h\|x-y\|, \quad \forall x, y, z \in X,
$$

and

$$
\lambda_{g}+\lambda_{H} \lambda_{g}+\lambda \lambda_{N_{1}} \delta_{A}+\lambda \lambda_{N_{2}} \delta_{B}+\lambda \lambda_{N_{3}} \delta_{C}<[\xi(1+r)-h] .
$$

Then, there exist $x \in X, u \in A(x), v \in B(x)$ and $w \in C(x)$ such that variational inclusion problem (8) is solvable. Moreover, $x_{n} \rightarrow x, u_{n} \rightarrow u, v_{n} \rightarrow v$ and $w_{n} \rightarrow w$, as $n \rightarrow \infty$, where $\left\{x_{n}\right\},\left\{u_{n}\right\},\left\{v_{n}\right\}$ and $\left\{w_{n}\right\}$ are the sequences defined in iterative Algorithm 3.1. 
Proof

Using the strong monotonicity of $g$ with constant $\xi$, we have

$$
\begin{aligned}
\left\|g\left(x_{n+1}\right)-g\left(x_{n}\right)\right\|\left\|x_{n+1}-x_{n}\right\| & \geq\left\langle g\left(x_{n+1}\right)-g\left(x_{n}\right), x_{n+1}-x_{n}\right\rangle \\
& \geq \xi\left\|x_{n+1}-x_{n}\right\|^{2}
\end{aligned}
$$

which implies that

$$
\left\|x_{n+1}-x_{n}\right\| \leq \frac{1}{\xi}\left\|g\left(x_{n+1}\right)-g\left(x_{n}\right)\right\| .
$$

By iterative Algorithm 3.1, Theorem 2.2 and condition (16), we have

$$
\begin{aligned}
& \left\|g\left(x_{n+1}\right)-g\left(x_{n}\right)\right\| \\
= & \| R_{\lambda, M\left(., x_{n}\right)}^{I-H}\left[(I-H) g\left(x_{n}\right)-\lambda N\left(u_{n}, v_{n}, w_{n}\right)\right]-R_{\lambda, M\left(., x_{n-1}\right)}^{I-H}\left[(I-H) g\left(x_{n-1}\right)\right. \\
& \left.-\lambda N\left(u_{n-1}, v_{n-1}, w_{n-1}\right)\right] \| \\
= & \| R_{\lambda, M\left(., x_{n}\right)}^{I-H}\left[(I-H) g\left(x_{n}\right)-\lambda N\left(u_{n}, v_{n}, w_{n}\right)\right]-R_{\lambda, M\left(., x_{n}\right)}^{I-H}\left[(I-H) g\left(x_{n-1}\right)\right. \\
& \left.-\lambda N\left(u_{n-1}, v_{n-1}, w_{n-1}\right)\right]+R_{\lambda, M\left(., x_{n}\right)}^{I-H}\left[(I-H) g\left(x_{n-1}\right)\right. \\
& \left.-\lambda N\left(u_{n-1}, v_{n-1}, w_{n-1}\right)\right]-R_{\lambda, M\left(., x_{n-1}\right)}^{I-H}\left[(I-H) g\left(x_{n-1}\right)-\lambda N\left(u_{n-1}, v_{n-1}, w_{n-1}\right)\right] \| \\
\leq & \| R_{\lambda, M\left(., x_{n}\right)}^{I-H}\left[(I-H) g\left(x_{n}\right)-\lambda N\left(u_{n}, v_{n}, w_{n}\right)\right] \\
& -R_{\lambda, M\left(., x_{n}\right)}^{I-H}\left[(I-H) g\left(x_{n-1}\right)-\lambda N\left(u_{n-1}, v_{n-1}, w_{n-1}\right)\right]\|+\| R_{\lambda, M\left(., x_{n}\right)}^{I-H}\left[(I-H) g\left(x_{n-1}\right)\right. \\
& \left.-\lambda N\left(u_{n-1}, v_{n-1}, w_{n-1}\right)\right]-R_{\lambda, M\left(., x_{n-1}\right)}^{I-H}\left[(I-H) g\left(x_{n-1}\right)-\lambda N\left(u_{n-1}, v_{n-1}, w_{n-1}\right)\right] \| \\
\leq \quad & \frac{1}{[1+r]}\left\|(I-H) g\left(x_{n}\right)-\lambda N\left(u_{n}, v_{n}, w_{n}\right)-\left((I-H) g\left(x_{n-1}\right)-\lambda N\left(u_{n-1}, v_{n-1}, w_{n-1}\right)\right)\right\| \\
& +h\left\|x_{n}-x_{n-1}\right\| .
\end{aligned}
$$

Since $g$ is Lipschitz continuous with constant $\lambda_{g}, H$ is a Lipschitz continuous with constant $\lambda_{H}, N$ is Lipschitz continuous in all three arguments with constants $\lambda_{N_{1}}, \lambda_{N_{2}}, \lambda_{N_{3}}$, respectively and $A, B, C$ are $D$-Lipschitz continuous with constants $\delta_{A}, \delta_{B}$ and $\delta_{C}$, respectively, we have

$$
\begin{aligned}
& \left\|(I-H) g\left(x_{n}\right)-(I-H) g\left(x_{n-1}\right)-\lambda\left(N\left(u_{n}, v_{n}, w_{n}\right)-N\left(u_{n-1}, v_{n-1}, w_{n-1}\right)\right)\right\| \\
\leq & \left\|(I-H) g\left(x_{n}\right)-(I-H) g\left(x_{n-1}\right)\right\|+\lambda\left\|N\left(u_{n}, v_{n}, w_{n}\right)-N\left(u_{n-1}, v_{n-1}, w_{n-1}\right)\right\| \\
\leq & \left\|g\left(x_{n}\right)-g\left(x_{n-1}\right)\right\|+\left\|H\left(g\left(x_{n}\right)\right)-H\left(g\left(x_{n-1}\right)\right)\right\|+\lambda \| N\left(u_{n}, v_{n}, w_{n}\right)- \\
& N\left(u_{n-1}, v_{n}, w_{n}\right)+N\left(u_{n-1}, v_{n}, w_{n}\right)+N\left(u_{n-1}, v_{n-1}, w_{n}\right)-N\left(u_{n-1}, v_{n-1}, w_{n}\right) \\
& -N\left(u_{n-1}, v_{n-1}, w_{n-1}\right) \|
\end{aligned}
$$




$$
\begin{aligned}
\leq & \left\|g\left(x_{n}\right)-g\left(x_{n-1}\right)\right\|+\left\|H\left(g\left(x_{n}\right)\right)-H\left(g\left(x_{n-1}\right)\right)\right\|+\lambda \| N\left(u_{n}, v_{n}, w_{n}\right)+ \\
& N\left(u_{n-1}, v_{n}, w_{n}\right)\|+\lambda\| N\left(u_{n-1}, v_{n}, w_{n}\right)-N\left(u_{n-1}, v_{n-1}, w_{n}\right) \|+ \\
& \lambda\left\|N\left(u_{n-1}, v_{n-1}, w_{n}\right)-N\left(u_{n-1}, v_{n-1}, w_{n-1}\right)\right\| \\
\leq & \lambda_{g}\left\|x_{n}-x_{n-1}\right\|+\lambda_{H} \lambda_{g}\left\|x_{n}-x_{n-1}\right\|+\lambda \lambda_{N_{1}}\left\|u_{n}-u_{n-1}\right\|+ \\
& \lambda \lambda_{N_{2}}\left\|v_{n}-v_{n-1}\right\|+\lambda \lambda_{N_{3}}\left\|w_{n}-w_{n-1}\right\| \\
\leq & \lambda_{g}\left\|x_{n}-x_{n-1}\right\|+\lambda_{H} \lambda_{g}\left\|x_{n}-x_{n-1}\right\|+\lambda \lambda_{N_{1}} D\left(A\left(x_{n}\right), A\left(x_{n-1}\right)\right)+ \\
& \lambda \lambda_{N_{2}} D\left(B\left(x_{n}\right), B\left(x_{n-1}\right)\right)+\lambda \lambda_{N_{3}} D\left(C\left(x_{n}\right), C\left(x_{n-1}\right)\right) \\
\leq & \lambda_{g}\left\|x_{n}-x_{n-1}\right\|+\lambda_{H} \lambda_{g}\left\|x_{n}-x_{n-1}\right\|+\lambda \lambda_{N_{1}} \delta_{A}\left\|x_{n}-x_{n-1}\right\|+ \\
& \lambda \lambda_{N_{2}} \delta_{B}\left\|x_{n}-x_{n-1}\right\|+\lambda \lambda_{N_{3}} \delta_{C}\left\|x_{n}-x_{n-1}\right\| \\
\leq & {\left[\lambda_{g}+\lambda_{H} \lambda_{g}+\lambda \lambda_{N_{1}} \delta_{A}+\lambda \lambda_{N_{2}} \delta_{B}+\lambda \lambda_{N_{3}} \delta_{C}\right]\left\|x_{n}-x_{n-1}\right\| . }
\end{aligned}
$$

Using (20), (19) becomes

$$
\left\|g\left(x_{n+1}\right)-g\left(x_{n}\right)\right\| \leq\left[\frac{\left[\lambda_{g}+\lambda_{H} \lambda_{g}+\lambda \lambda_{N_{1}} \delta_{A}+\lambda \lambda_{N_{2}} \delta_{B}+\lambda \lambda_{N_{3}} \delta_{C}\right]}{[1+r]}+h\right]\left\|x_{n}-x_{n-1}\right\| .
$$

Using (21), (18) becomes

$$
\left\|x_{n+1}-x_{n}\right\| \leq \theta\left\|x_{n}-x_{n-1}\right\|
$$

where

$$
\theta=\frac{1}{\xi}\left[\frac{\left.\lambda_{g}+\lambda_{H} \lambda_{g}+\lambda \lambda_{N_{1}} \delta_{A}+\lambda \lambda_{N_{2}} \delta_{B}+\lambda \lambda_{N_{3}} \delta_{C}\right]}{[1+r]}+h\right] .
$$

By condition (17), we have $0 \leq \theta<1$, thus $\left\{x_{n}\right\}$ is a Cauchy sequence in $X$ and as $X$ is complete, there exists $x \in X$ such that $x_{n} \rightarrow x$ as $n \rightarrow \infty$. From (13), (14) and (15) of Algorithm 3.1 and $D$-Lipschitz continuity of $A, B$ and $C$ with constants $\delta_{A}, \delta_{B}$ and $\delta_{C}$, respectively, we have

$$
\begin{gathered}
\left\|u_{n+1}-u_{n}\right\| \leq D\left(A\left(x_{n+1}\right), A\left(x_{n}\right)\right) \leq \delta_{A}\left\|x_{n+1}-x_{n}\right\| ; \\
\left\|v_{n+1}-v_{n}\right\| \leq D\left(B\left(x_{n+1}\right), B\left(x_{n}\right)\right) \leq \delta_{B}\left\|x_{n+1}-x_{n}\right\| ; \\
\left\|w_{n+1}-w_{n}\right\| \leq D\left(C\left(x_{n+1}\right), C\left(x_{n}\right)\right) \leq \delta_{C}\left\|x_{n+1}-x_{n}\right\| .
\end{gathered}
$$

It is clear from (23), (24) and (25) that $\left\{u_{n}\right\},\left\{v_{n}\right\}$ and $\left\{w_{n}\right\}$ are also Cauchy sequences in $X$, so there exist $u, v$ and $w$ in $X$ such that $u_{n} \rightarrow u, v_{n} \rightarrow v$ and $w_{n} \rightarrow w$. By using the continuity of the operators $I, N, M, g, A, B, C$, $H, R_{\lambda, M(., x)}^{I-H}$ and iterative Algorithm 3.1, we have

$$
g(x)=R_{\lambda, M(., x)}^{I-H}[(I-H) g(x)-\lambda N(u, v, w)] .
$$

By Lemma 3.1, we conclude that $(x, u, v)$ is a solution of problem (3.1). It remains to show that $u \in A(x), v \in B(x)$ and $w \in C(x)$. In fact

$$
\begin{aligned}
d(u, A(u)) & \leq\left\|u-u_{n}\right\|+d\left(u_{n}, A(x)\right) \\
& \leq\left\|u-u_{n}\right\|+D\left(A\left(x_{n}\right), A(x)\right) \\
& \leq\left\|u-u_{n}\right\|+\delta_{A}\left\|x_{n}-x\right\| \rightarrow 0, \text { as } n \rightarrow \infty .
\end{aligned}
$$

Hence $u \in A(x)$. Similarly, we can show that $v \in B(x)$ and $w \in C(x)$. This completes the proof. 


\section{Conclusion}

The resolvent operator techniques are applicable to solve several problem related to variational inequalities (inclusions), optimization problems, complementary problems etc..The aim of this work is to introduce a new type of resolvent operator based on relaxed Lipschitz continuity and monotonicity, and we call it as relaxed resolvent operator. We prove that relaxed resolvent operator is single valued and Lipschitz continuous. We define an iterative algorithm to approximate the solution of a variational inclusion problem. In our opinion, many other problems occurring in applied sciences may be solved by using relaxed resolvent operator in a different frame work.

\section{REFERENCES}

1. C. Baiocchi, A. Capelo: Variational and quasi-variational inequalities, John Wiley and Sons, New York (1984).

2. S.S. Chang: Set-valued variational inclusions in Banach spaces, Applied Mathematics and Computation, Vol. 248, pp. 438-454 (2000).

3. S.S. Chang, Y.-J. Cho, B.S. Lee and I.H. Jung: Generalized set-valued variational inclusions in Banach spaces, Applied Mathematics and Computation, Vol. 246, pp. 409-422 (2000).

4. S.S. Chang, J.K. Kim and K.H. Kim: On the existence and iterative approximation problems of solutions for set-valued variational inclusions in Banach spaces, Applied Mathematics and Computation, Vol. 268, pp. 89-108 (2002).

5. Y.P. Fang and N.-J. Huang: H-accretive operator and resolvent operator technique for variational inclusions in Banach spaces, Applied Mathematics Latters, Vol. 17, No. 6, pp. 647-653 (2004).

6. Y.P. Fang and N.-J. Huang: $H$-monotone operator and resolvent operator technique for variational inclusions, Applied Mathematics and Computation, Vol. 145, No. 2-3, pp. 795-803 (2003).

7. Y.P. Fang, Y.-J. Chu, and J.K. Kim: $(H, \eta)$-accretive operator and approximating solutions for systems of variational inclusions in Banach spaces, to appear in Applied Mathematics Letters.

8. Y.P. Fang and N.-J. Huang: Approximate solutions for non-linear variational inclusions with $(H, \eta)$-monotone operator, Research Report, Sichuan University (2003).

9. Y.P. Fang, N.-J. Huang, and H.B. Thompson: A new system of variational inclusions with $(H, \eta)$-monotone operators in Banach spaces, Computers \& Mathematics with Applications, Vol. 49, No. 2-3, pp. 365-374 (2005).

10. A. Hassouni and A. Moudafi: A perturbed algorithm for variational inclusions, Journal of Mathematical Analysis and Application, Vol. 183(3), pp. 706-712 (1994).

11. K.R. Kazmi, F.A. Khan: Iterative approximation of a solution of multi-valued variational-like inclusion in Banach spaces: A P- $\eta$ proximal-point mapping approach, Journal of Mathematical Analysis and Application, Vol. 325, pp. 665-674 (2007).

12. D. Kinderlehrer, G. Stampacchia: An introduction to variational inequalities and their applications, Academic Press, New York (1980).

13. H.-Y. Lan, Y.J. Chu and R.U. Verma: Non-linear relaxed cocoercive variational inclusions involving $(A, \eta)$-accertive mappings in Banach spaces, Computers \& Mathematics with Applications, Vol. 51, No. 9-10, pp. 1529-1538 (2006).

14. Jr.S.B. Nadler: Multivalued contraction mappings, Pacific Journal of Mathematics, Vol 30, pp. 475-488 (1992).

15. A. Nagurney: Network economics: A variational inequality approach, Kluwer Academic Publishers, Dordrecht (2002).

16. M.A. Noor, K.I. Noor and R. Kamal: General variational inclusions involving defference of operators, Journal of Inequalities and Applications, 2014. 2014:98.

17. M. Patriksson: Non-linear programming and variational inequality problems: A unified approach, Kluwer Academic Publishers, Dordrecht (1999).

18. A.H. Siddiqi and Q.H. Ansari: An algorithm for a class of quasi-variational inequalities, Journal of Mathematical Analysis and Application, Vol. 145(2), pp. 413-418 (1990).

19. Y.K. Tang, S.S. Chang and S. Salahuddin: A system of nonlinear set-valued variational inclusions, Springer Plus, (2014) doi: 10.1186/2193-1801-3-318.

20. S. Wang: On fixed point and variational inclusion problems, Filomat, Vol. 29(6), pp. 1409-1417 (2015)

21. D.L. Zhu and P. Marcotte: Co-coercivity and its role in the convergence of iterative schemes for solving variational inequalities, SIAM Journal of Optimization, Vol 6, No. 3, pp. 714-726 (1996). 